\title{
Reticulocyte Volume Distribution Width Coefficient of Variation
}

National Cancer Institute

\section{Source}

National Cancer Institute. Reticulocyte Volume Distribution Width Coefficient of Variation. NCl Thesaurus. Code C139072.

A measurement of the volume dispersion within a reticulocyte population, calculated as the standard deviation of the mean reticulocyte volume divided by the mean reticulocyte volume, multiplied by 100 for conversion to a percentage. 